\title{
REVISIÓN DE TEMA
}

Residente de Medicin

Física y Rehabilitación, Facultad de Medicina, Escuela de posgrados, Universidad de la Sabana, Chía, Cundinamarca, Colombia

2Especialista en Medicina física y rehabilitación, profesor clínico, Facultad de Medicina, Universidad de la Sabana, Chía, Cundinamarca, Colombia

${ }^{3}$ Especialista en Medicina física y rehabilitación. Clínica Universidad de La

Sabana, profesor clínico, coordinador de la especialidad en Medicina física y

rehabilitación. Facultad de Medicina, Universidad de la Sabana, Chía, Cundinamarca, Colombia

${ }^{4}$ Semiólogo, especialista en Lenguaje y Producción Intelectual Científica. Docente de Investigación. Universidad Javeriana, Facultad de Medicina, Universidad de la Sabana, Chía, Cundinamarca, Colombi

Recibido:

5 de marzo de 2018

Aceptado

16 de abril de 2018

Correspondencia:

David Leonardo Laverde davidlaag@unisabana.edu.co

Conflictos de interés: No tiene conflicto de interés.

\section{Educación médica en rehabilitación en pregrado: Un reto en el mundo Medical education in rehabilitation in undergraduate: a world challenge}

David Leonardo Laverde Aguirre1, Liliana Margarita García Gutiérrez², Catalina Gómez Guevara3 ${ }^{3}$ William Ángel Salazar ${ }^{4}$

\section{RESUMEN}

Propósito: La medicina en rehabilitación hace una importante contribución al cuidado de la salud de una gran población y en forma integral encuentra formas de ayudar a pacientes incluso cuando los procesos quirúrgicos se agotan. En ese contexto, aporta medios para minimizar el impacto de las condiciones discapacitantes y alcanzar un nivel de autonomía e interacción normal en la sociedad. Por lo tanto, se hace necesario que los médicos, tanto generales como de todas las especialidades, que tratan pacientes con enfermedades crónicas y limitaciones funcionales, reciban formación y capacitación en las facultades de medicina en los principios del manejo y la rehabilitación de enfermedades agudas, subagudas y crónicas. El presente artículo analiza y describe a través de evidencia el estado actual de la educación médica en rehabilitación, específicamente en la formación de pregrado.

Métodos: Revisión de la literatura del periodo 2004-2018 (14 años), en publicaciones relacionadas con educación médica en rehabilitación.

Conclusión: Existen muchos intentos de implementar y evaluar programas curriculares de educación en rehabilitación, los resultados son variables y muestran que aún se encuentra en continua investigación. En Colombia no se encuentran estudios que indaguen acerca de los diferentes programas de educación en rehabilitación. Es necesario promover la investigación en docencia médica en esta rama de la medicina.

Palabras clave: Educación de pregrado en medicina, medicina física y rehabilitación, evaluación educacional.

DOI: http://dx.doi.org/10.28957/rcmfr.v28n1a6

\section{ABSTRACT}

Purpose: Medicine in rehabilitation makes an important contribution to the health care of a large population; in an integral way, he finds ways to help patients when surgical procedures are exhausted. In this context, it provides means to minimize the impact of disabling conditions and achieve a level of autonomy and normal interaction in society. Therefore, it is necessary that in general physicians, general practitioners and specialist, which are related to chronic diseases and functional disabilities, receive training in medical schools in the principles of management and the rehabilitation of acute diseases, subacute and chronic. This article analyzes and describes through the evidence the current state of medical education in rehabilitation, specifically in undergraduate education.

Methods: Review of the literature of the period 2004-2018 (14 years), in publications related to medical education in rehabilitation.

Conclusion: There are many attempts to implement and evaluate curricular programs of education in rehabilitation, the results are variable and it is a field that is still in continuous investigation. In Colombia there are no studies that inquire about the different education programs in rehabilitation. It is necessary to promote research in medical teaching in this branch of medicine.

Key words: Medical education in undergraduate, Physical medicine and rehabilitation, educational measurement.

DOI: http://dx.doi.org/10.28957/rcmfr.v28n1a6 


\section{INTRODUCCIÓN}

La Medicina Física y Rehabilitación (en adelante MF\&R) es la rama de la medicina especializada en el diagnóstico, la prevención y el tratamiento de múltiples desórdenes neurológicos y músculo-esqueléticos; proporciona intervenciones en rehabilitación, especialmente en aquellos pacientes con dificultades físicas y cognitivas. Tiene una visión holística del paciente y potencial para reducir su discapacidad tanto en el paciente ambulatorio como en el hospitalizado ${ }^{1}$.

Durante la educación médica en pregrado los estudiantes son expuestos a diferentes áreas del conocimiento médico a través de materias y rotaciones en cada área específica. Lo anterior logra dar, además de conocimiento, una perspectiva acerca de la utilidad de cada una de ellas dentro del ámbito médico. En Colombia, en la descripción del perfil del graduado en medicina general, se incluye el proceso y las competencias de rehabilitación ${ }^{2}$; sin embargo, no existe norma que genere una obligación a las escuelas de medicina para incluir educación en medicina física y rehabilitación en sus diferentes estructuras curriculares.

Aún más, es frecuente que los médicos graduados no reconozcan, incluso, la existencia de la MF\& R como especialidad médica; tampoco su campo de acción. Lo anterior limita y retarda el acceso por parte de los pacientes a la atención por rehabilitación. En el periodo 2004-2018, la investigación sobre educación médica en rehabilitación ha aumentado a partir de múltiples intentos por reglamentar su implementación teniendo en cuenta la importancia de la discapacidad de una población adulta que sobrevive en mayor medida a enfermedades catastróficas y traumas severos.

En ese sentido, la educación médica en rehabilitación ha tomado relevancia y ha aumentado el interés en el ámbito académico médico, lo que hace pertinente identificar su situación actual en diferentes países y, en especial, en Colombia, lo cual permitirá tomar decisiones sobre una mayor implementación o acciones de mejora. Por lo tanto, el objetivo central de esta revisión es describir el estado reciente de la investigación en docencia médica relevante a la MF\& R en el pregrado médico. Para tal efecto, se realizó una revisión en Pubmed para los artículos que contuvieran las palabras clave (medical education, rehabilitation, students) durante 2004-2018, con el fin de obtener los artículos que correspondieran a educación médica en rehabilitación; adicionalmente, se filtraron los artículos en inglés y en español. Se encontraron 654 artículos, de los cuales se revisaron los títulos y se seleccionaron 22 como objeto de esta revisión, seleccionados por incluir alguna de las palabras usadas y contar con enfoque al temario objeto de la revisión; se descartaron aquellos artículos que no correspondieran a educación y a medicina y que no evaluaran los programas académicos. Estos estudios no solo resaltan los problemas asociados a la rehabilitación; también presentan modelos e intentos por mejorar la enseñanza en rehabilitación.

\section{CONCEPTOS}

La discapacidad es un concepto dinámico que se encuentra en constante evolución; es el resultado no solo del individuo, sino también de su interacción con su entorno. En la actualidad la OMS utiliza el enfoque biopsicosocial para la definición y enfoque de la discapacidad. No obstante, existe evidencia de que la discapacidad en ocasiones ha sido vista desde la óptica de las creencias religiosas, siendo considerada como un castigo divino o una condición moral; eseto en detrimento de esta población ${ }^{3}$. La legislación colombiana establece en su política pública nacional de discapacidad e inclusión social (PPDIS), que se deben asegurar los derechos y deberes de forma plena de las personas con discapacidad, sus familias y cuidadores en el periodo 2013-20224.

De manera adicional, por parte del Ministerio de Protección Social se establece el con- 
cepto de la rehabilitación integral en Colombia, cuyo objeto es facilitar la promoción, prevención, recuperación, rehabilitación e inclusión social de la población ${ }^{5}$. La Rehabilitación es definida como: «El uso de todos los medios encaminados a la reducción de las condiciones de discapacidad y desventajas, habilitando a las personas con discapacidad para alcanzar su nivel máximo de integración social» 6 . Por esto, es de vital importancia el contacto de todo el personal médico hacia este concepto. Esto es más importante si se tiene en cuenta que aumenta el número de población en condición de discapacidad por causa de la inversión de la pirámide poblacional y el aumento de la supervivencia para las enfermedades catastróficas y los traumas severos. Todo lo anterior ha aumentado el número de personas con problemas complejos y déficit funcionales ${ }^{6}$.

\section{EDUCACIÓN EN REHABILITACIÓN}

La revisión permitió encontrar diferentes estudios encaminados a establecer cómo se aborda la educación médica en rehabilitación en diferentes partes del mundo; los estudios intentan establecer el nivel de conciencia por parte de los estudiantes de medicina acerca de la discapacidad, las metodologías de enseñanza y los cambios necesarios por realizar. La población en situación de discapacidad representa la más grande minoría poblacional? .

A pesar de que el conocimiento en Medicina de Rehabilitación es ampliamente considerado como una competencia básica para el estudiante de medicina, y el médico general es quien más contacto tiene con la población en situación de discapacidad, se observa que la educación en esta área es limitada ${ }^{8}$. El conocimiento sobre el concepto de discapacidad y la rehabilitación, de forma general, no hace parte de la educación médica de pregrado, por lo que cuenta con poca intensidad horaria y un contenido ineficiente en su enseñanza9 ${ }^{9}$. Es claro que el médico de forma constante, indiferente a su especialidad, se enfrenta en su práctica médica al paciente en situación de discapacidad de cualquier grado ${ }^{10}$; también se ve enfrentado al requerimiento de rehabilitación y la prescripción de terapias; en consecuencia, es prioritario que todos los médicos adquieran un conocimiento básico acerca de la medicina física y rehabilitación $(M F \& R)^{11}$. Entre otras habilidades que son dominio de la MF\& R se encuentra el conocimiento del sistema nervioso periférico y central y el sistema músculo-esquelético, lo cual es de enorme utilidad para la formación del médico general ${ }^{12}$.

Sin embargo, la MF\& R no ha sido importante entre los estudiantes de medicina ${ }^{13}$; en Colombia no todos los programas de medicina establecen una rotación en rehabilitación dentro de su estructura curricular. Pero este no es un problema que solo afecte a Colombia; en otros países la percepción de la especialidad en MF\& $R$ también se ve impactada por la falta de una constante para el establecimiento de una rotación en MF\& R dentro del pregrado $^{12}$. Así, solo el $52 \%$ de 75 escuelas de medicina en Estados Unidos que fueron evaluadas, contaban con un programa que incluía concientización acerca de la discapacidad ${ }^{7}$. Se evidencia que la intensidad horaria a la cual los médicos en formación están expuestos es altamente variable, con algunos datos que muestran que en otros países la oferta de este entrenamiento puede ser tan alta como 12/13 escuelas de medicina, por ejemplo, en Polonia ${ }^{6}$, sin cumplir un número de horas adecuado para este entrenamiento. La medicina en rehabilitación en los países desarrollados no ha tenido un avance como otras áreas de la medicina, dadas sus características de tener una naturaleza única, la inversión a largo plazo, el trabajo en equipo y el trabajo con los más necesitados ${ }^{14}$.

De igual manera, se encontró que este déficit no solo se presenta en pregrado, sino también en otras áreas de la medicina. Así, se encontraron valores tan altos como un 47,3\% de los médicos, quienes consideran que tienen un entrenamiento inadecuado para el cuidado en rehabilitación ${ }^{15}$. 


\section{ESTADO ACTUAL}

Existen esfuerzos de investigación para evaluar el impacto de la educación en MF\& R dentro del programa de medicina de pregrado ${ }^{13}$. Se ha demostrado que incluso rotaciones cortas, con objetivos claros, mejoran el conocimiento y la actitud de los estudiantes de pregrado acerca de la MF\& R ${ }^{16}$. La sociedad europea de medicina física y rehabilitación y la sociedad británica de medicina de rehabilitación (ESPRM y BSRM por sus siglas en inglés, respectivamente) han desarrollado estándares para enseñanza médica en rehabilitación a los estudiantes de medicina ${ }^{13}$. Estos son ${ }^{13}$ :

- Los principios de la rehabilitación y el modelo biopsicosocial de la clasificación internacional del funcionamiento, discapacidad y salud (CIF).

- La organización y práctica del sistema de rehabilitación (rehabilitación aguda y post aguda, así como también programas de rehabilitación para pacientes con condiciones crónicas).

- Los principios y el potencial de las áreas terapéuticas (fisioterapia, terapia ocupacional y otras áreas afines).

- Programas integrales de rehabilitación y sus principales indicaciones.

- Las necesidades de rehabilitación de los pacientes con afecciones específicas (por ejemplo, accidente cerebrovascular, politraumatismo, dolor lumbar, artritis, cáncer, etc.).

- Conocimiento del sistema de seguridad social y la legislación concerniente a la discapacidad y la reintegración, en nivel nacional, así como los aspectos éticos y humanos en rehabilitación.

A pesar de que la investigación en educación médica es valiosa, generalmente es ignorada ${ }^{8}$, aun cuando es importante, para obtener líneas de base que permitan el posterior desarrollo de programas de educación. Las escuelas de medicina que no cuentan con un programa de formación en conocimiento encaminado hacia la población con discapacidad presentan barreras para su implementación, llamando sorprendentemente la atención que el obstáculo más común es que no existe nadie defendiendo la implementación de esta área en el currículo. La segunda barrera identificada fue la restricción de tiempo en cuanto a la representación de la especialidad en el programa de la carrera. Lo anterior teniendo en cuenta la vasta cantidad de información de todas las áreas que debe ser organizada para un programa de pregrado. Como tercera barrera se encuentra la falta de recursos para la implementación para el desarrollo?

La falta de oportunidades de exposición a la especialidad conlleva a que se subestime la especialidad, lo que a su vez ocasiona una presencia académica baja en las escuelas de medicina, siendo un ciclo que disminuye la calidad de los programas en rehabilitación ${ }^{13}$. Figura 1.

\section{ESFUERZOS PARA LA EDUCACIÓN EN REHABILITACIÓN}

Se han realizado varios estudios donde se evaluaron las intervenciones en la educación en medicina de rehabilitación, a través de entrevistas a estudiantes, médicos residentes y especialistas. Se encontró la realización de una feria de interés para promover el conocimiento en el área de la MF\& ${ }^{17}$, llevando a los estudiantes a exponerse de forma directa a la especialidad, contando con posterior evaluación a la exposición en la feria. Este estudio reportó logros importantes en cuanto al aumento de interés hacia la MF\& R y la posibilidad de realizar demostraciones e interacciones hacia las diferentes áreas de conocimiento implicadas con la rehabilitación; se destacó que el área con mayor interés fuera el uso de dispositivos médicos, más específicamente la ultrasonografía y la medicina electrodiagnóstica ${ }^{17}$. En ese sentido, se resalta la importancia de lograr la exposición de los estudiantes con sus futuros intereses y la posi- 


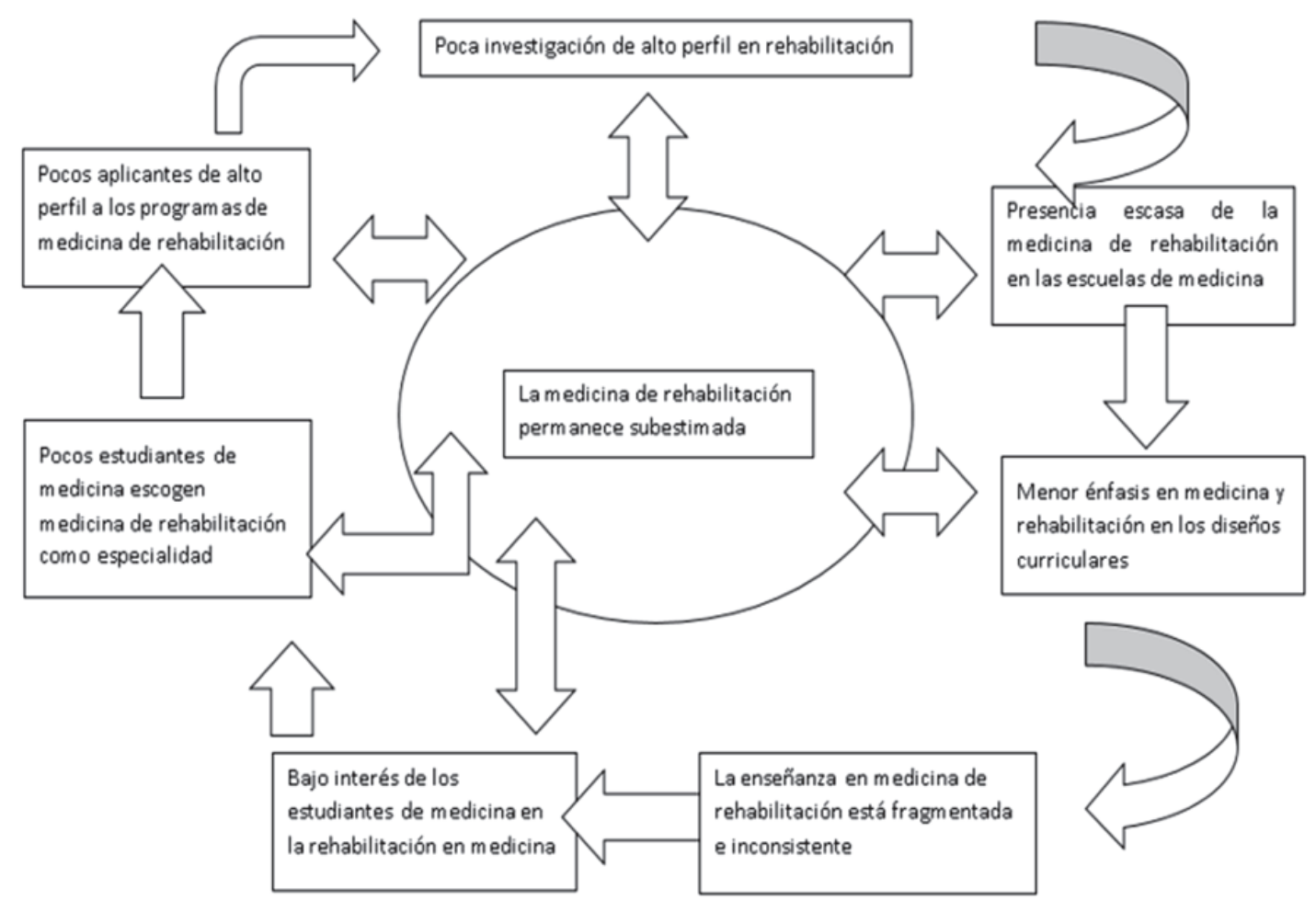

Figura 1. Perfil potencial de la medicina de rehabilitación en las escuelas médicas. Tomado de Teaching medical students rehabilitation medicine. ${ }^{13}$

bilidad de conocer mentores que guíen a través de esta afinidad con la especialidad de MF\& $\mathrm{R}^{17}$.

En Alemania, la escuela de medicina de Hannover realizó un estudio con el esfuerzo para integrar un curso de rehabilitación en el currículo médico ${ }^{11}$, cuyos principales objetivos eran introducir los principios de rehabilitación de forma tan pronta como fuera posible y profundizar en el trabajo con entrenamiento práctico en MF\&R. De manera adicional, también daban la posibilidad de que aquellos estudiantes con especial interés en el área tomaran electivas con relación a esta área de conocimiento. La división propuesta por este modelo de Hannover incluyó lecturas, talleres y entrenamiento práctico, además de una división por áreas de interés de la especialidad, a saber: introducción a la especialidad, rehabilitación y medicina física. La evaluación a este modelo muestra una buena aceptación, con el requerimiento de una continua evaluación de la docencia ${ }^{11}$.

Pero la MF\& R no solamente se requiere como rotación; además, brinda conocimientos en áreas relevantes para la formación médica, principalmente en cuanto a las habilidades para el examen físico músculo-esquelético. Considerando que cada día será mayor la población con más de 65 años, con desórdenes osteomusculares, contando hasta con 4 de las 10 primeras causas en consulta según publicaciones españolas ${ }^{25}$, el desarrollo de una rotación, guiada por médicos de rehabilitación, logra un impacto positivo en cuanto al conocimiento y el desempeño de sus participantes en la evaluación músculo-esquelética ${ }^{18}$. Esto resalta el importante papel de la MF\&R en la formación de profesionales médicos.

El requerimiento de estas rotaciones como parte del currículo tiene un impacto positivo en el nivel de conocimiento, por parte de los estudiantes, acerca de la especialidad, no siendo sorpresivo este resultado dado que en forma general la exposición es baja a esta área médica ${ }^{16}$. Dadas las limitaciones previas de tiempo para la inclusión de este tipo de formación a nivel de rehabilitación, se han liderado propuestas para tener componentes online ${ }^{15}$, ferias de interés ${ }^{17}$ y la inclusión dentro de otros 
cursos $^{12}$. Todo lo anterior orientado a lograr la mayor exposición del estudiante.

\section{DISCUSIÓN}

El objetivo de esta revisión fue indagar el estado actual de la educación médica en rehabilitación; se revisó a través de la literatura de varios países y se encontró una tendencia en aumento del interés de las diferentes escuelas acerca de la integración de la especialidad en los currículos de los pregrados de medicina. Muchos autores admiten que incluir la MF\& R en los programas médicos es un deber y una oportunidad para aumentar el tratamiento integral, aun cuando las posibilidades quirúrgicas sean eliminadas. De aquí se deriva la importancia de incluir un conocimiento mayor, puesto que aún hay muchas especialidades afines con la MF\& R que no están sensibilizadas acerca de esta especialidad ${ }^{6}$. En general, se observó la inadecuada formación de los estudiantes de medicina en cuanto a rehabilitación, lo que exige un conocimiento y entrenamiento en la MF\& R, más aún cuando aporta de manera importante al entrenamiento médico general, especialmente en las áreas de evaluación y abordaje del sistema músculo-esquelético, condiciones crónicas y rehabilitación. Por lo tanto, se destaca que en la actualidad la educación médica continuada es una fuente importante de información tanto para médicos rehabilitadores, como para el resto de médicos y más aún un conocimiento base integral de formación médica ${ }^{6}$.

Los esfuerzos han logrado rotaciones con módulos teórico-prácticos que se encuentran en rangos entre una y cuatro semanas; sin embargo, es necesaria la implementación de un concepto sistemático integrado para la enseñanza de la rehabilitación ${ }^{11}$. En ese sentido, es vital para lograr avances en su enseñanza y aplicación una continua evaluación de los contenidos, medición del impacto de las rotaciones y las intervenciones realizadas, lo que permitiría una mayor educación en rehabilitación.

Existen muchas oportunidades para que los estudiantes aprendan y se expongan a la medi- cina de rehabilitación, pero estas oportunidades no son siempre explícitas o formalmente usadas ${ }^{19}$. No hay un consenso acerca del nivel de formación en el cual se debe realizar esta exposición, es decir, son variables los niveles en los cuales se realizan las intervenciones en los estudios revisados; esto ha permitido reconocer las habilidades que brinda una rotación en MF\& R, tanto para un médico general como para un especialista; también se acepta que forman parte de una adecuada formación médica. Adicionalmente, es de vital importancia intervenir en las habilidades del personal encargado de transmitir estos conocimientos.

Se han propuesto modelos de educación que se encuentran en prueba e implementación (tabla 1). No obstante, es importante aclarar que la presente revisión está limitada por el periodo de búsqueda, dado que las revisiones clásicas acerca del tema son reducidas y de la década de los noventa, lo que implica la necesidad de ampliar las investigaciones y estudios sobre el tema. Otra limitante es la concentración de estudios en inglés, pues no se presentan revisiones en español, ni otros idiomas diferentes.

\section{CONCLUSIÓN}

En definitiva, la MF\& $R$ es una especialidad médica que permite a los médicos en formación, habilidades clínicas y conocimientos importantes en forma transversal para su práctica cotidiana, principalmente en las áreas de manejo de la discapacidad y patologías del sistema músculo-esquelético; esto quiere decir que es una de las formas directas para ampliar el conocimiento, las habilidades y competencias de un médico integral, necesario en la formación moderna de este profesional.

Es crítico que en la educación en pregrado existan bajos niveles de conocimiento acerca de la discapacidad y sus estudios, con baja o ausente exposición a la MF\& R respecto a otras especialidades médicas. En el periodo 2002 2017 el estudio de la educación médica en rehabilitación a nivel mundial ha ido en aumento, 
Tabla 1. Sugerencias para mejorar la enseñanza en medicina de rehabilitación

- Introducir MF\& R de forma temprana en los programas de pregrado.

- Asegurar adecuadas bases de las sesiones de enseñanza.

- Considerar la participación de terapeutas y enfermeras en el personal de enseñanza.

- Involucrar pacientes en la enseñanza.

- Involucrar pacientes en el desarrollo del curso.

- Desarrollar una base de datos de pacientes expertos para las sesiones de enseñanza.

- Considerar el uso de la CIF como un marco de enseñanza.

- Mantener la enseñanza magistral y las lecturas a un mínimo.

- Múltiples sesiones prácticas cortas (ej. $45 \mathrm{~min}$ ); mantener un número bajo de estudiantes para cada sesión, garantizar tiempos de desplazamientos cortos, contacto con pacientes cara a cara, juegos de roles y videos de habilidades funcionales de pacientes con discapacidades.

- Incluir diagnóstico y fisiopatología en el ámbito del cuidado primario.

- Integrar la enseñanza de rehabilitación con otras especialidades relevantes; por ejemplo, reumatología, ortopedia, neurología, neurocirugía.

- Asegurar que el programa esté lleno con pocas brechas.

- Fuerte presencia médica en sesiones de enseñanza, pero con sesiones de equipo multidisciplinario.

- Enseñanza centrada en los tipos comunes de discapacidad.

- Enfatizar en la evaluación funcional en la enseñanza del examen físico.

- Incorporar evaluaciones en los cursos (ej. cuestionarios de opciones múltiples, examen clínico objetivado).

- Incluir retroalimentación por parte de los estudiantes en el desarrollo del curso.

- No desalentarse por pobre retroalimentación.

Ejemplo de propuesta de aspectos a mejorar de la educación en rehabilitación (adaptado de Gibson et al., 201013).

pero aun así no hay datos claros en Colombia acerca de este fenómeno, lo que exige impulsar investigaciones que permitan un conocimiento mayor.

Aunque se han propuesto modelos para diferentes tipos de intervenciones en la enseñanza en rehabilitación, además de la necesidad de realizar una continua evaluación de las reformas realizadas a los diferentes programas educativos, en Colombia no se encuentran estudios que describan la enseñanza en rehabilitación.

Esta revisión abre paso a temáticas de estudio necesarias en el país, con el fin de mejorar la calidad de la educación y hacer de la MF\&R un área de la medicina conocida de una forma más amplia, garantizando así mayor investigación, calidad de la formación a todos los niveles y futuro de la especialidad.

Finalmente, se recomienda realizar estudios donde se establezca cómo es el estado actual de la educación en rehabilitación en los diferentes programas de pregrado en medicina en el país, además de la posibilidad de evaluar cómo impacta el conocimiento en MF\& R en la práctica de los médicos generales y de especialistas en diferentes ramas.

\section{CONTRIBUCIÓN DE LOS AUTORES}

William Ángel Salazar P.: Docente investigador, aporte en redacción, revisión de la estructura del escrito.

Dra. Catalina Gómez: Médica fisiatra, consultora temática.

Dra. Liliana García: Médica fisiatra, consultora temática, revisión de estructura del artículo, redacción.

Dr. David Leonardo Laverde: Médico fisiatra, revisión temática, revisión y estructura del artículo.

\section{RESPONSABILIDADES ÉTICAS}

\section{Confidencialidad de los datos}

Los autores declaran que han seguido los protocolos de su centro de trabajo sobre la publicación de datos de pacientes.

\section{Divulgación de conflicto de intereses}

Los autores no tienen ningún conflicto de intereses para declarar. 


\section{REFERENCIAS}

1. Raeissadat S, Samadi B, Rayegani S, Bahrami M, Mahmoudi H. Survey of Medical Residents' Attitude Toward Physical Medicine and Rehabilitation. American Journal of Physical Medicine \& Rehabilitation. 2014;93(6):540-547.

2. Consultorsalud.com. (2018). Competencias del médico de Colombia 2012. Disponible en: http://www.consultorsalud.com/sites/ consultorsalud/files/Competencias_del_ medico_de_Colombia_2012.pdf

3. Couser G. What Disability Studies Has to Offer Medical Education. Journal of Medical Humanities. 2010;32(1):21-30.

4. Colombia, M. (2018). Política Pública Nacional de Discapacidad e Inclusión Social. Minsalud.gov.co Disponible en: https:// www.minsalud.gov.co/proteccionsocial/ promocion-social/Discapacidad/Paginas/ politica-publica.aspx

5. Colombia, M. (2018). Discapacidad. [online] Minsalud.gov.co. Disponible en: https:// www.minsalud.gov.co/proteccionsocial/Paginas/DisCAPACIDAD.aspx

6. Tederko, P., Krasuski, M., Denes, Z., Moslavac, S. and Likarevic, I. (2018). What medical doctors and medical students know about physical medicine and rehabilitation: a survey from central Europe. European Journal of physical and rehabilitation Medicine, 52(5), pp.597-605.

7. Seidel E, Crowe S. The State of Disability Awareness in American Medical Schools. American Journal of Physical Medicine \& Rehabilitation. 2017;96(9):673-676.

8. Raissi G, Mansoori K, Madani P, Rayegani $\mathrm{S}$. Survey of general practitioners? attitudes toward physical medicine and rehabilitation. International Journal of Rehabilitation Research. 2006;29(2):167-170.

9. Tonko V, Mladen B, Nadija F, Reuben E. Teaching Disability and Rehabilitation Medicine at the Medical School in Split, Croatia. Croatian Medical Journal. 2004;45(1):99-102.
10. Sarmiento C, Miller S, Chang E, Zazove P, Kumagai A. From Impairment to Empowerment. Academic Medicine. 2016;91(7): 954-957.

11. Gutenbrunner C, Schiller J, Schwarze M, Fischer V, Paulmann V, Haller $\mathrm{H}$ et al. Hannover model for the implementation of physical and rehabilitation medicine teaching in undergraduate medical training. Journal of Rehabilitation Medicine. 2010;42(3):206-213.

12. Newcomer K, Laskowski E, Grande J, Dyrbye L. The Physiatrists' Crucial Role in the Development and Implementation of a Longitudinal Musculoskeletal Physical Examination Curriculum in a Medical School. American Journal of Physical Medicine \& Rehabilitation. 2013;92(1):84-89.

13. Gibson J, Lin X, Clarke K, Fish H, Phillips M. Teaching medical students rehabilitation medicine. Disability and Rehabilitation. 2010;32(23):1948-1954.

14. Reza Raissi G, Vahdatpour B, Ashraf A, Mansouri K. Integrating physical medicine and rehabilitation into the curriculum of Iranian medical students. Disability and Rehabilitation. 2006;28(1):67-70.

15. Mayer R, Shah A, DeLateur B, Durso S. Proposal for a Required Advanced Clerkship in Chronic Disease and Disability for Medical Students. American Journal of Physical Medicine \& Rehabilitation. 2008;87(2):162-167.

16. Faulk C, Mali J, Mendoza P, Musick D, Sembrano R. Impact of a Required FourthYear Medical Student Rotation in Physical Medicine and Rehabilitation. American Journal of Physical Medicine \& Rehabilitation. 2012;91(5):442-448.

17. Brane, L., Carson, R., Susmarski, A., Lewno, A. and Dicianno, B. (2017). Changing Perception. Outcomes from a Physical Medicine and Rehabilitation Medical Student Interest Fair. American Journal of Physical Medicine \& Rehabilitation, 96(5), pp. 362-365.

18. Altschuler E, Cruz E, Salim S, Jani J, Stitik T, Foye P et al. Efficacy of a Checklist as Part of 
a Physical Medicine and Rehabilitation Clerkship to Teach Medical Students Musculoskeletal Physical Examination Skills. American Journal of Physical Medicine \& Rehabilitation. 2014;93(1):82-89.

19. Graham S, Eley D, Cameron I, Thistlethwaite J. Inclusion of rehabilitation medicine concepts in school of medicine resources. Disability and Rehabilitation. 2013;36(18): 1555-1561.

20. Jones P, Donald M. Teaching medical students about children with disabilities in a rural setting in a school. BMC Medical Education. 2007;7(1).

21. Brane L, Carson R, Susmarski A, Lewno A, Dicianno B. Changing Perception. American Journal of Physical Medicine \& Rehabilitation. 2017;96(5):362-365.

22. Campbell F. Medical Education and Disability Studies. Journal of Medical Humanities. 2009;30(4):221-235.

23. Couser G. What Disability Studies Has to Offer Medical Education. Journal of Medical Humanities. 2010;32(1):21-30.
24. Moroz A, Gonzalez-Ramos G, Festinger T, Langer K, Zefferino S, Kalet A. Immediate and follow-up effects of a brief disability curriculum on disability knowledge and attitudes of PM\& $R$ residents: A comparison group trial. Medical Teacher. 2010;32(8): e360-e364.

25. Vlak T, Soso D, Poljicanin A, Becir B, Marinovic I, Pivalica D et al. Physical and rehabilitation medicine training center in Split, Croatia: striving to achieve excellence in education of a rehabilitation team. Disability and Rehabilitation. 2013;36(9): 781-786.

26. Los problemas de salud más frecuentes en atención primaria [Internet]. Siete Días Médicos. Revista de atención primaria. 2018 [cited 15 February 2018]. Disponible en: http://www.sietediasmedicos.com/actualidad/en-portada/item/6348-los-problemasde-salud-mas-frecuentes-en-atencion-primaria\#. WoWopejOXIU 